\title{
Pequenos mamíferos não-voadores (Didelphimorphia, Rodentia) em campos litorâneos do extremo sul do Brasil
}

\author{
Fernando Marques Quintela ${ }^{1,5}$, Bruno Igure Gonçalves ${ }^{2}$, Gabriel Eberts Trindade ${ }^{2}$, \\ Maurício Beux dos Santos ${ }^{3}$ \& Alexandro Marques Tozetti ${ }^{4}$ \\ 'Programa de Pós-graduação em Biologia Animal, Instituto de Biociências, \\ Universidade Federal do Rio Grande do Sul - UFRGS, Av. Bento Gonçalves, 9500, \\ CEP 91501-970, Porto Alegre, RS, Brasil. http://www.ufrgs.br \\ ${ }^{2}$ Laboratório de Ecologia de Vertebrados Terrestres, Instituto de Ciências Biológicas, \\ Universidade Federal do Rio Grande - FURG, Campus Carreiros, Av. Itália, Km 8, \\ CEP 96201-900, Rio Grande, RS, Brasil. http://www.furg.br \\ ${ }^{3}$ Programa de Pós-graduação em Biodiversidade Animal, Centro de Ciências Naturais e Exatas, \\ Universidade Federal de Santa Maria - UFSM, Av. Roraima, s/n, Camobi, \\ CEP 97105-900, Santa Maria, RS, Brasil. http://www.ufsm.br \\ ${ }^{4}$ Universidade do Vale do Rio dos Sinos - UNISINOS, Av. Unisinos, 950, \\ CEP 93022-000, São Leopoldo, RS, Brasil. http://www.unisinos.br \\ ${ }_{5}^{5}$ Autor para correspondência: Fernando Marques Quintela, e-mail: fmquintela@yahoo.com.br
}

QUINTELA, F.M., GONÇALVES, B.I., TRINDADE, G.E., SANTOS, M.B. \& TOZETTI, A.M. Non-volant small mammals (Didelphimorphia, Rodentia) in coastal grasslands of southernmost Brazil. Biota Neotrop. 13(4): http://www.biotaneotropica.org.br/v13n4/en/abstract?inventory+bn03013042013

\begin{abstract}
Non-volant small mammals are key elements in natural environments due its importance as food resource. This study aimed to investigate the species composition and habitat occupancy by non-volant small mammals (Didelphimorphia, Rodentia) in a coastal grassland area in southern Rio Grande do Sul coastal plain. Between April 2009 and March 2010 pitffal traps were used to capture small mammals in coastal grasslands associated to sand dunes and arbustive Restinga. We sampled 180 individuals including two marsupials (Cryptonanus guahybae and Didelphis albiventris) and seven rodent species (Calomys laucha, Cavia aperea, Ctenomys flamarioni, Deltamys kempi, Oligoryzomys flavescens, Oxymycterus nasutus and Scapteromys tumidus). Cryptonanus guahybae, D. albiventris andC. flamarioni were captured only in dunes while C. aperea and O. nasutus were recorded only in arbustive Restinga habitats. Calomys laucha, D. kempi, O. flavescens and O. nasutus were captured in both habitats. Oligoryzomys flavescens and C. laucha were the most representative species in both habitats, comprising respectively 40.7 and $38.9 \%$ of captures in dunes and 56.3 and $34.9 \%$ of captures in Restinga habitats. The species richness recorded in the sampled coastal grasslands area was lower than those previously recorded in subtropical forest coastal systems.
\end{abstract}

Keywords: coastal environments, marsupials, rodents, Calomys laucha, Oligoryzomys flavescens, assemblage.

QUinTElA, F.M., GONÇAlves, B.I., TRINDADE, G.E., SANTOS, M.B. \& TOZETTI, A.M. Pequenos mamíferos não-voadores (Didelphimorphia, Rodentia) em campos litorâneos do extremo sul do Brasil. Biota Neotrop. 13(4): http://www.biotaneotropica.org.br/v13n4/pt/abstract?inventory+bn03013042013

Resumo: Pequenos mamíferos não-voadores são elementos chave em ambientes naturais devido a sua importância como recurso alimentar. Este estudo teve como objetivo investigar a composição de espécies e a ocupação de habitat por pequenos mamíferos não-voadores (Didelphimorphia, Rodentia) em uma área de campos litorâneos na região sul da planície costeira do Rio Grande do Sul. Entre abril de 2009 e março de 2010 armadilhas de interceptação e queda foram utilizadas para a captura de pequenos mamíferos em campos associados a dunas e a restinga arbustiva. Foram amostrados 180 indivíduos pertencentes a duas espécies de marsupiais (Cryptonanus guahybae e Didelphis albiventris) e sete espécies de roedores (Calomys laucha, Cavia aperea, Ctenomys flamarioni, Deltamys kempi, Oligoryzomys flavescens, Oxymycterus nasutus e Scapteromys tumidus). Cryptonanus guahybae, D. albiventris e C. flamarioni foram capturados somente em dunas, enquanto que $C$. aperea e $O$. nasutus foram registrados somente em restinga arbustiva. Calomys laucha, D. kempi, O. flavescens e O. nasutus foram capturados em ambos os ambientes. Oligoryzomys flavescens e C. laucha foram as espécies mais representativas em ambos os ambientes, compreendendo respectivamente 40,7 e 38,9\% das capturas em dunas, e 56,3 e 34,9\% das capturas em restinga. A riqueza de espécies registrada na área de campo litorâneo amostrada foi inferior a aquelas previamente registradas em sistemas florestais costeiros subtropicais.

Palavras-chave: ambientes costeiros, marsupiais, roedores, Calomys laucha, Oligoryzomys flavescens, assembléia. 


\section{Introdução}

Pequenos mamíferos são elementos chave em comunidades naturais, atuando em diversos níveis tróficos e nos processos de predação e dispersão de sementes (Moura et al. 2008). Neste contexto, inventários focando assembléias desses organismos são a base para a compreensão de processos ecológicos tais como dinâmica de populações e estruturas de comunidades e para o desenvolvimento de planos de manejo em ambientes naturais (Moura et al. 2008).

No Rio Grande do Sul, a maioria dos inventários com ênfase em pequenos mamíferos não-voadores foi conduzida em formações florestais tais como Floresta com Araucária (Floresta Ombrófila Mista) (Cademartori et al. 2002, 2004, Dalmagro \& Vieira 2005, Iob \& Vieira 2008), ecótono Floresta com Araucária-Campo (Pedó 2005), Floresta Atlântica sensu stricto (Floresta Ombrófila Densa) (Horn 2005) e florestas de restinga (Oliveira 1985, Langone 2007, Quintela et al. 2012, Sponchiado et al. 2012). Entretanto, muito pouco se conhece sobre a composição e riqueza de espécies em dunas costeiras e ambientes associados.

As dunas costeiras, no Brasil, representam ecossistemas típicos de bacias sedimentares, denominados "restingas", formados durante eventos de transgressão e regressão marinha do Holoceno (Araújo \& Lacerda 1987). Devido à alta drenagem, escassez de nutrientes e efeito de dessecação do spray salino, as dunas costeiras têm sido consideradas desertos edáficos, onde os movimentos da areia, os níveis de umidade, o input salino, a vegetação e os microclimas são elementos chave para a ocorrência e distribuição de espécies animais (McLachlan 1991). Os campos litorâneos ou mantos de dispersão eólica são resultantes da erosão eólica sobre as dunas costeiras (Vieira 1984). Nos mantos estabilizados, as sucessivas etapas de colonização da vegetação acabam por criar um habitat mais estruturado, desenvolvendo-se aí formações de restinga herbácea/ subarbustiva, arbustiva e arbórea (Waechter 1985, Falkenberg 1999). Apesar das condições extremas, estudos recentes têm revelado níveis consideráveis de riqueza específica de vertebrados nesses sistemas, incluindo anfíbios (Oliveira et al. 2013) e répteis (Santos et al. 2012).

Estudos sobre pequenos mamíferos em ambientes litorâneos do Rio Grande do Sul são restritos a determinadas espécies como Ctenomys flamarioni Travi (Ctenomyidae) (e.g. Gava \& Freitas 2003, Freitas 2006, Fernandes et al. 2007) e Calomys laucha G. Fischer (Camargo et al. 2006). Aspectos como composição de assembléias, padrões de abundância e densidade, área de vida, uso do habitat e microhabitat por marsupiais e roedores em dunas costeiras permanecem pouco conhecidos. O objetivo deste trabalho é determinar a riqueza e a composição de espécies de pequenos mamíferos não-voadores (marsupiais e pequenos roedores) em uma área de campos litorâneos associados a dunas costeiras e restingas arbustivas no extremo sul do Brasil.

\section{Material e Métodos}

\section{1. Área de estudo}

Este estudo foi conduzido em uma área de aproximadamente $0.42 \mathrm{~km}^{2}$ de campos litorâneos situados na Praia do Cassino ( $32^{\circ} 07^{\prime}$ 54 " S e $52^{\circ} 20^{\prime} 53^{\prime}$ W), município de Rio Grande, Rio Grande do Sul (Figura 1). O clima na região é classificado como subtemperado úmido, com temperatura media anual de $18,1^{\circ} \mathrm{C}$, precipitação média anual de $1.162 \mathrm{~mm}$ e estações bem definidas (Maluf 2000). Os meses mais chuvosos são julho, agosto e setembro (Vieira 1983).

Foram amostrados dois ambientes distintos: (1) campos úmidos associados ao cordão de dunas costeiras (designados como "dunas costeiras"). Solo em grande parte arenoso exposto, com predominância gramíneo/herbácea, sendo representativas Panicum racemosum Spr. e Senecio crassiflorus (Lam.) DC. (2) campos associados a formações arbustivas de restinga (denominados "restingas arbustivas"). Formação arbustiva/arbórea com alta representatividade de Lithraea brasiliensis Marchand e Chrysophyllum marginatum (Hook \& Arn.) (Santos et al. 2013), e substrato composto por serapilheira sobre solo arenoso. A primeira formação está localizada mais próxima à praia oceânica em relação à segunda.

\section{Amostragens}

As coletas foram realizadas entre abril de 2009 e março de 2010. Os pequenos mamíferos foram capturados em armadilhas de interceptação e queda constituídas por quatro baldes de 100 litros conectados por uma cerca-guia de tela plástica (malha $1,45 \mathrm{~mm}$ ) de $40 \mathrm{~m}$ de comprimento e $0,50 \mathrm{~m}$ de altura. As cercas-guia foram enterradas cerca de $10 \mathrm{~cm}$ no substrato, trespassando os baldes nesta mesma profundidade. Os baldes foram dispostos linearmente em cada armadilha. Cada estação de captura compreendeu duas armadilhas. Três estações espaçadas por aproximadamente 600 $\mathrm{m}$ foram instaladas em cada ambiente, totalizando 12 armadilhas, $480 \mathrm{~m}$ de cerca-guia e 48 baldes. O espaçamento entre as estações foi estabelecido com o objetivo de se obter uma maior distribuição dos esforços de amostragem sobre os ambientes, considerando-se a possibilidade de ocorrência de espécies de baixa vagilidade. Os baldes permaneceram abertos por cinco dias consecutivos em períodos quinzenais, totalizando 120 dias de amostragem e um esforço de captura de 5.760 baldes/dia e noite. Os baldes foram revisados diariamente durante os períodos de amostragem.

Todos os indivíduos foram coletados e identificados a partir da análise de caracteres externos e crânio-dentários. Crânios e peles foram depositados na coleção de mamíferos do Museu de Ciências Naturais da Universidade Luterana do Brasil (ULBRA), Canoas, Rio Grande do Sul (MCNU). A nomenclatura taxonômica utilizada segue Paglia et al. (2012). As coletas foram autorizadas pelo Instituto Chico Mendes (ICMBio; processo no. 29358-1).

\section{Resultados}

Foram capturados 180 indivíduos pertencentes a duas espécies de marsupiais (Cryptonanus guahybae Tate e Didelphis albiventris Lund) e sete espécies de roedores (Calomys laucha, Cavia aperea Erxleben, Ctenomys flamarioni, Deltamys kempi Thomas, Oligoryzomys flavescens Waterhouse, Oxymycterus nasutus Waterhouse e Scapteromys tumidus Waterhouse). As duas espécies de marsupiais e cinco espécies de roedores foram registradas em ambientes de dunas, enquanto seis espécies de roedores foram registradas em ambientes de restinga (Tabela 1). Em ambos os ambientes Oligoryzomys flavescens foi a espécie com maior número de capturas, seguida por Calomys laucha (Figura 2). O sucesso de captura foi 1,84\% em dunas costeiras e $4,40 \%$ em restinga arbustiva.

\section{Discussão}

A riqueza de espécies $(n=9)$ encontrada na área de campos litorâneos amostrada no presente estudo foi inferior a aquelas registradas na maioria dos inventários previamente realizados em outros ecossistemas do Brasil subtropical, a exemplo de Cherem \& Perez (1996) $(\mathrm{n}=11)$, Horn (2005) $(\mathrm{n}=11)$, Pedó (2005) $(\mathrm{n}=13)$, Graipel et al. (2006) $(n=11)$, Langone (2007) $(n=10)$, Quintela et al. (2012) $(n=11)$. Este fato pode ser um reflexo da menor complexidade estrutural dos ambientes de campo litorâneo quando comparados às formações florestais e mosaicos ambientais onde os estudos supramencionados foram realizados. Uma correlação positiva entre riqueza de espécies de pequenos mamíferos não-voadores e 

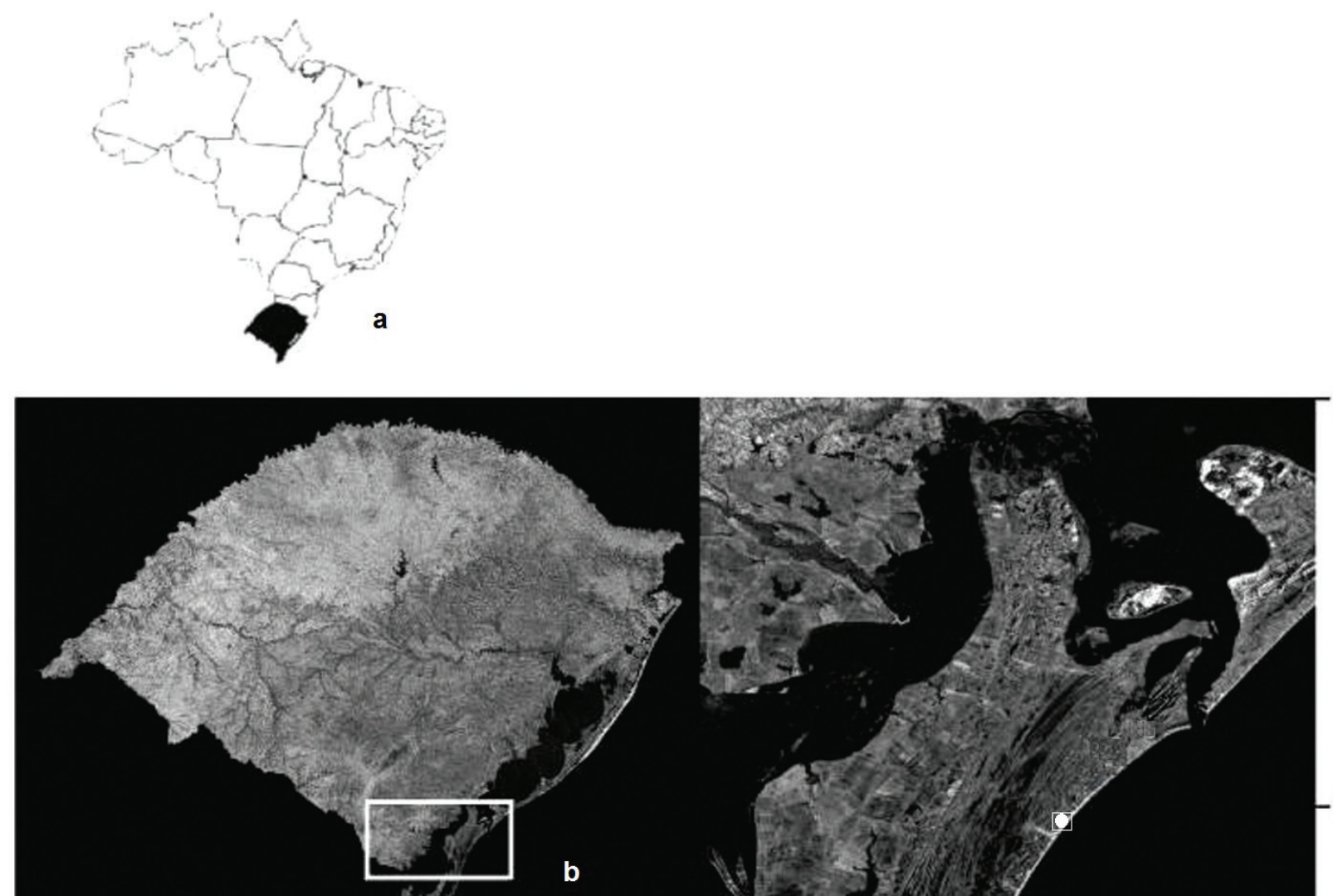

$31^{\circ} 45$ 's
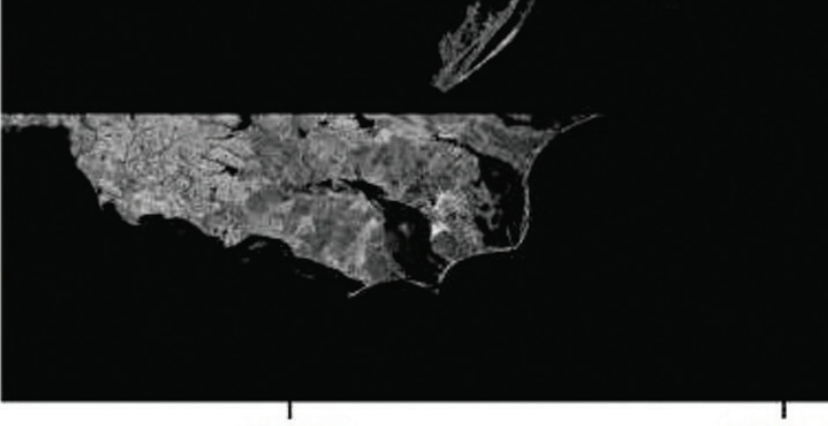

$53^{\circ} 15^{\prime} \mathrm{O}$

$52^{\circ} 45^{\prime} \mathrm{O}$

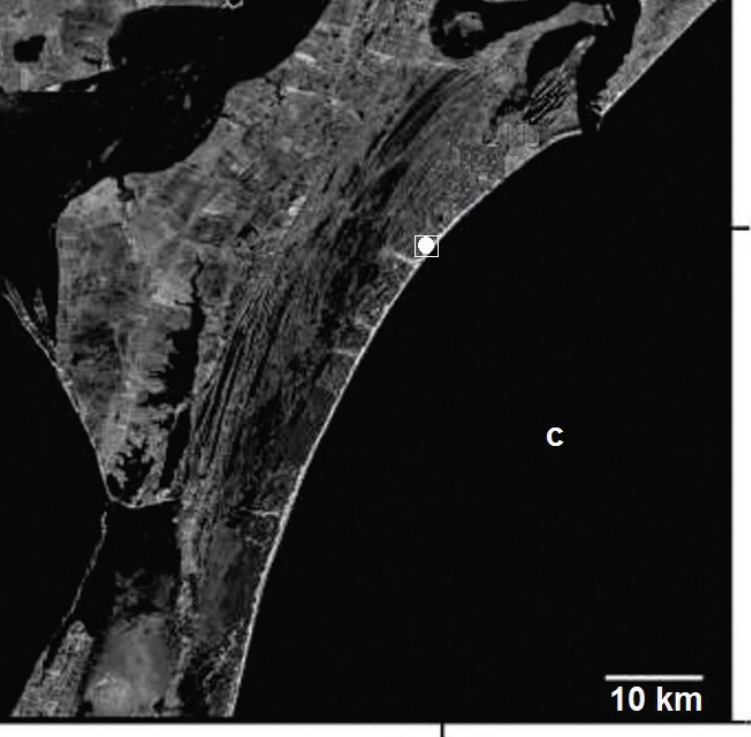

Figura 1. Localização do Estado do Rio Grande do Sul (a), região sul da Planície Costeira (b) e área de estudo (c).

Figure 1. Location of state of Rio Grande do Sul State (a), southern Coastal Plain (b) and study area (c).

complexidade de habitat já foi demonstrada em diversas investigações em ecossistemas tropicais e subtropicais (e.g. August 1983, Fonseca 1989, Kerley 1992, Lyra Jorge et al. 2001). Cabe também ressaltar a diferença entre os métodos de captura utilizados no presente estudo (armadilhas de interceptação e queda) e nos estudos precedentes (live traps), o que pôde ter contribuído para as dissimilaridades observadas entre a riqueza e a composição de espécies.

De acordo com McLachlan (1991), a riqueza de espécies faunísticas em dunas costeiras está relacionada a diferenças na vegetação e complexidade de habitat ao longo de um gradiente perpendicular à praia oceânica. No presente estudo, uma riqueza similar foi registrada nos dois ambientes amostrados, apesar das diferenças estruturais entre os dois sistemas. Entretanto, diferenças quanto à composição de espécies foram observadas entre os dois ambientes. Ctenomys flamarioni é endêmico das dunas frontais (Fernandes et al. 2007) e Calomys laucha tem associação com habitats abertos (González \& Lanfranco 2010). Todas as demais espécies já foram registradas tanto em fisionomias abertas quanto em formações florestais no bioma Pampa (Langone 2007, González \& Lanfranco 2010, Quintela et al. 2012, Sponchiado et al. 2012). Apesar de não terem sido registradas, é provável que Cryptonanus guahybae e Didelphis albiventris também ocorram em campo litorâneo associado à restinga arbustiva. Cryptonanus guahybae já foi capturado em matas de restinga com alta densidade de indivíduos arbóreos (Quintela et al. 2011, 2012), enquanto D. albiventris é uma espécie generalista quanto ao habitat, comumente encontrada tanto em áreas abertas quanto em formações florestais (González \& Lanfranco 2010). Deltamys kempi, Oxymycterus nasutus e Scapteromys tumidus são relacionadas a áreas abertas úmidas e alagadas (Emmons \& Feer 1997, González \& Pardiñas 2002, González \& Lanfranco 2010), sendo que a presença destas espécies nas fisionomias arbustivas pode indicar a influência de sistemas palustres das áreas abertas do entorno, tais como banhados e campos úmidos adjacentes. A presença destas três espécies de Akodontini em ambientes de restinga pode também estar relacionada 
Pequenos mamíferos em campos litorâneos

Tabela 1. Número de indivíduos capturados (N) e porcentagem relativa de capturas (\%) das espécies de pequenos mamíferos não-voadores em dois ambientes de uma área de campos litorâneos no município de Rio Grande, Estado Rio Grande do Sul, Brasil.

Table 1. Number of captured individuals $(\mathrm{N})$ and relative percentage of captures (\%) of non-volant small mammals in two environments of a coastal grassland area in Rio Grande County, state of Rio Grande do Sul, Brazil.

\begin{tabular}{|c|c|c|c|c|}
\hline \multirow{2}{*}{ Taxon } & \multicolumn{2}{|c|}{ Dunas } & \multicolumn{2}{|c|}{ Restinga } \\
\hline & $\mathbf{N}$ & $\%$ & $\mathbf{N}$ & $\%$ \\
\hline \multicolumn{5}{|l|}{ Didelphimorpia } \\
\hline \multicolumn{5}{|l|}{ Didelphidae } \\
\hline Cryptonanus guahybae (Tate, 1931) & 1 & 1,8 & & \\
\hline Didelphis albiventris Lund, 1840 & 1 & 1,8 & & \\
\hline \multicolumn{5}{|l|}{ Rodentia } \\
\hline \multicolumn{5}{|l|}{ Cricetidae } \\
\hline Calomys laucha (Fischer, 1914) & 21 & 38,9 & 44 & 34,9 \\
\hline Deltamys kempi (Thomas, 1817) & 6 & 11,1 & 9 & 7,1 \\
\hline Oligoryzomys flavescens (Waterhouse, 1837) & 22 & 40,7 & 71 & 56,3 \\
\hline Scapteromys tumidus (Waterhouse, 1837) & 1 & 1,8 & 1 & 0,07 \\
\hline Oxymycterus nasutus Waterhouse, 1837 & & & 1 & 1,8 \\
\hline \multicolumn{5}{|l|}{ Caviidae } \\
\hline Cavia aperea Erxleben, 1777 & & & 1 & 0,07 \\
\hline \multicolumn{5}{|l|}{ Ctenomyidae } \\
\hline Ctenomys flamarioni Travi, 1981 & 1 & 1,8 & & \\
\hline Total & 53 & 100 & 127 & 100 \\
\hline Riqueza de espécies & 7 & & 6 & \\
\hline
\end{tabular}
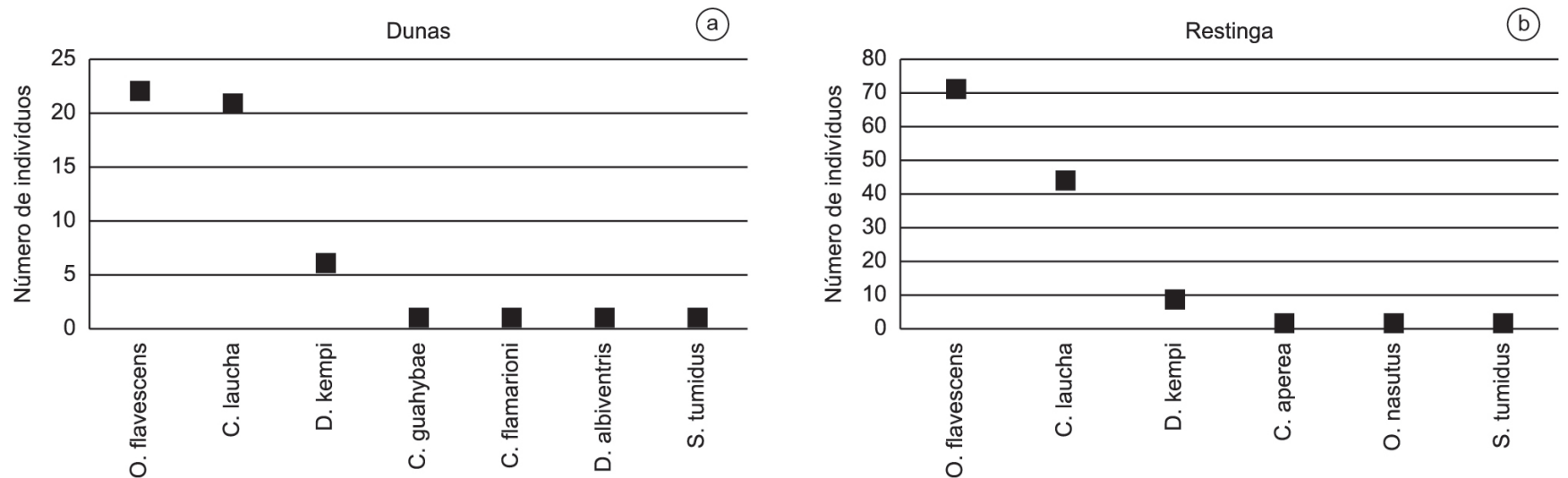

Figura 2. Rank de espécies de pequenos mamíferos não-voadores registrados em campos litorâneos associados a dunas (a) e restinga (b), município de Rio Grande, Estado do Rio Grande do Sul, Brasil.

Figure 2. Rank of species of non-volant small mammals recorded in coastal fields associated to dunes (a) and Restinga (b), Rio Grande County, state of Rio Grande do Sul, Brazil.

à densa cobertura de serapilheira e consequente disponibilidade de artrópodes, itens predominantes na dieta destas espécies (González \& Lanfranco 2010).

Oligoryzomys flavescens e Calomys laucha foram as espécies numericamente dominantes em ambos os ambientes amostrados. Oligoryzomys flavescens já foi registrada em uma variedade de habitats no Rio Grande do Sul, incluindo formações campestres (Oliveira 1985, Pedó 2005, Langone 2007, Sponchiado et al. 2012), banhado (Langone 2007), Floresta Ombrófila Mista (Marinho 2003, Dalmagro \& Vieira 2005), floresta palustre (Oliveira 1985, Marinho 2003, Quintela et al. 2012) e floresta arenosa ciliar (Quintela et al. 2012). Já Calomys laucha esteve ausente na maioria dos estudos precedentes, sendo registrada por Oliveira (1985) em área campestre. Isso sugere que $C$. laucha, possa ser uma espécie fortemente associada aos campos litorâneos na região sul da planície costeira do Rio Grande do Sul. Outras espécies com ocorrência assinalada para a região sul da Planície Costeira do Rio Grande do Sul são o didelfídeo Lutreolina crassicaudata Desmarest, os sigmodontíneos Akodon azarae G. Fischer, A. reigi González, Langguth \& Oliveira, Brucepattersonius iheringi Thomas, Holochilus brasiliensis Desmarest, H. vulpinus Brants e Wilfredomys oenax Thomas e os histricomorfos Cavea magna Ximenez e Ctenomys torquatus Lichtenstein (Oliveira 1985, Fernandes et al. 2007, Bonvicino et al. 2008, Gava et al. 2012, Quintela et al. 2012, Sponchiado et al. 2012). É possível, portanto, que tais espécies ocorram em áreas de campo litorâneo, nos ambientes de maior adequabilidade.

O presente estudo revelou que a área de campos litorâneos amostrada abriga $47 \%$ do total de espécies de pequenos mamíferos não-voadores registradas na região sul da Planície Costeira do Rio Grande do Sul (Oliveira 1985, Fernandes et al. 2007, Bonvicino et al. 
2008, Gava et al. 2012, Quintela et al. 2012, Sponchiado et al. 2012), o que reforça a importância da preservação deste ecossistema para a manutenção da diversidade da mastofauna regional. Foi observada uma dissimilaridade na riqueza e composição de espécies entre os dois ambientes amostrados, sendo o ambiente de dunas costeiras o que apresentou maior riqueza e os únicos registros de marsupiais.

\section{Agradecimentos}

Somo gratos a Eduardo Coelho e Alexandre U. Christoff pela preparação de crânios e peles dos espécimes coletados, aos revisores anônimos pelas correções e sugestões ao manuscrito e aos órgãos financiadores do projeto CNPq, FAPERGS e CAPES pelo apoio financeiro e pelas bolsas de pós-graduação concedidas.

\section{Referências Bibliográficas}

ARAÚJO, D.S.D. \& LACERDA, L.D. 1987. A natureza das restingas. Ciênc. Hoje 6(33):42-8.

AUGUST, P.V. 1983. The role of habitat complexity and heterogeneity in structuring tropical mammal communities. Ecol. 64(6):1495-1507. http:// dx.doi.org/10.2307/1937504

BONVICINO, C.R., OLIVEIRA, J.A. \& D'ANDREA, P.S. 2008. Guia dos roedores do Brasil, com chaves para gêneros baseadas em caracteres externas. Centro Pan-Americano de Febre Aftosa - OPAS/OMS, Rio de Janeiro.

CADEMARTORI, C.V., MARQUES, R.V., PACHECO, S.M., BAPTISTA, L.R.M. \& GARCIA, M. 2002. Roedores ocorrentes em Floresta Ombrófila Mista (São Francisco de Paula, Rio Grande do Sul) e a caracterização de seu hábitat. Com. Mus. Ciênc. Tecnol. PUCRS, Sér. Zool. 15(1):61-86.

CADEMARTORI, C.V., FÁBIAN, M.E. \& MENEGHETI, J.O. 2004. Variações na abundância de roedores (Rodentia, Sigmodontinae) em duas áreas de Floresta Ombrófila Mista, Rio Grande do Sul, Brasil. Rev. Bras. Zooc. 6(2):147-167.

CAMARgO, C.R., COlARES, E.P. \& CASTRUCCI, A.M.L. 2006. Seasonal pelage color change: news based on a South American rodent. An. Acad. Bras. Ciênc. 78(1):77-86. http://dx.doi.org/10.1590/S000137652006000100009

CHEREM, J.J. \& PEREZ, D.M. 1996. Mamíferos terrestres de floresta de araucária no município de Três Barras, Santa Catarina, Brasil. Biotemas 9(2):29-46.

DALMAGRO, A.D. \& VIEIRA, E.M. 2005. Patterns of habitat utilization of small rodents in an area of Araucaria forest in Southern Brazil. Austral Ecol. 30:353-362. http://dx.doi.org/10.1111/j.1442-9993.2005.01447.x

EMMONS, L.H. \& FEER, F. 1997. Neotropical Rainforest mammals - A field guide. The University of Chicago Press, Chicago.

FALKENBERG, D.B. 1999. Aspectos da flora e da vegetação secundária da restinga de Santa Catarina, Sul do Brasil. Insula 28:1-30.

FERNANDES, F.A., FERNÁNDEZ-STOLZ, G.P., LOPES, C.M. \& FREITAS, T.R.O. 2007. The conservation status of the tuco-tucos, genus Ctenomys (Rodentia: Ctenomyidae), in southern Brazil. Braz. J. Biol., 67(4, Suppl.):839-847. http://dx.doi.org/10.1590/S1519-69842007000500006

FONSECA, G.A.B. 1989. Small mammal species diversity in Brazilian tropical primary and secondary forests of different sizes. Revta. Bras. Zool. 6(3):381-422. http://dx.doi.org/10.1590/S0101-81751989000300001

FREITAS, T.R.O. 2006. Cytogenetics status of four Ctenomys species in the South of Brazil. Genetica (The Hague), 126(1):227-235.

GAVA, A. \& FREITAS, T.R.O. 2003. Inter and Intra-Specific Hybridization in Tuco-Tucos (Ctenomys) from Brazilian Coastal Plains (Rodentia: Ctenomyidae). Genetica (The Hague) 119:11-17.

GAVA, A., SANTOS, M.B. \& QUINTELA, F.M. 2012. A new karyotype for Cavia magna (Rodentia: Caviidae) from an estuarine island and $C$. aperea from adjacent mainland. Acta Theriol. 57:9-14. http://dx.doi. org/10.1007/s13364-011-0042-0
GONZÁLEZ, E.M. \& PARDIÑAS, F.J. 2002. Deltamys kempi. Mammal. Spec.771:1-4. http://dx.doi.org/10.1644/1545-1410(2002)711\%3C000 1:DK\%3E2.0.CO;2

GONZÁLEZ, E.M. \& LANFRANCO, J.A.M. 2010. Mamíferos de Uruguay. Guía de campo e introducción a su estúdio y conservación. Banda Oriental, Museo Nacional de Historia Natural, Vida Silvestre Uruguay, Montevideo.

GRAIPEL, M.E., CHEREM, J.J., MONTEIRO-FILHO, E.L.A. \& GLOCK, L. 2006. Dinâmica populacional de marsupiais e roedores no Parque Municipal da Lagoa do Peri, Ilha de Santa Catarina, Sul do Brasil. Mastozool. Neotrop. 13(1):31-49.

HORN, G.B. 2005. A assembléia de pequenos mamíferos da floresta paludosa do Faxinal, Torres-RS: sua relação com a borda e o roedor Akodon montensis (Rodentia, Muridae) como potencial dispersos de sementes endozoocóricas. Dissertação de mestrado, Universidade Federal do Rio Grande do Sul, Porto Alegre.

IOB, G. \& VIEIRA, E.M. 2008. Seed predation of Araucaria angustifolia (Araucariaceae) in the Brazilian Araucaria Forest: influence of deposition site and comparative role of small and 'large' mammals. Plant Ecol. 198:185-196. http://dx.doi.org/10.1007/s11258-007-9394-6

KERLEY, G.I.H. 1992. Ecological correlates of small mammal community structure in the semi-arid Karoo, South Africa. J. Zool. 227:17-27. http:// dx.doi.org/10.1111/j.1469-7998.1992.tb04341.x

LANGONE, P.Q. 2007. Importância da matriz e das características do habitat sobre a assembléia de pequenos mamíferos em fragmentos de restinga no sul do Brasil. Dissertação de mestrado, Universidade Federal do Rio Grande do Sul, Porto Alegre.

LYRA JORGE, M.C., PIVELLO, V.R., MEIRELLES, S.T. \& VIVO, M. 2001. Riqueza e abundância de pequenos mamíferos em ambientes de Cerrado e Floresta, na Reserva Cerrado do Pé-de-gigante, Parque Estadual de Vassununga (Santa Rita do Passa Quatro, SP). Naturalia 26:287-302.

MALUF, J.R.T. 2000. Nova classificação climática do Estado do Rio Grande do Sul. Rev. Bras. Agromet. 8(1):141-150.

MARINHO, J.R. 2003. Estudo da comunidade e do fluxo gênico de roedores silvestres em um gradiente altitudinal de Mata Atlântica na área de influência da RST-453/RS-486 - Rota do Sol. Tese doutorado, Universidade Federal do Rio Grande do Sul, Porto Alegre.

McLACHLAN, A. 1991. Ecology of coastal dune fauna. J. Arid Environ. 21: 229-243.

MOURA, M.C., GRELLE, C.E.V. \& BERGALLO, H.G. 2008. How does sampling protocol affect the richness and abundance of small mammals recorded in tropical forest? An example from the Atlantic Forest, Brazil. Neotrop. Biol. Conserv. 3(2):51-58.

OLIVEIRA, L.F.B. 1985. Estrutura e ordenação espaço-temporal de uma congregação de roedores no sul da Planície Costeira do Rio Grande do Sul, Brasil (Mammalia, Rodentia, Cricetidae). Dissertação de mestrado, Universidade Federal do Rio Grande do Sul, Porto Alegre.

OLIVEIRA, M.C.L.M., SANTOS, M.B., LOEBMANN, D. \& TOZETTI, A.M. 2013. Diversity and associations between coastal habitats and anurans in southernmost Brazil. An. Acad. Bras. Cienc. No prelo.

PAGLIA, A.P., FONSECA, G.A.B., RYLANDS, A.B., HERRMANN, G., AGUIAR, L.M.S., CHIARELLO, A.G., LEITE, Y.L.R., COSTA, L.P., SICILIANO, S., KIERULFF, M.C.M., MENDES, S.L., TAVARES, V.C., MITTERMEIER, R.A. \& PATTON, J.L. 2012. Lista Anotada dos Mamíferos do Brasil/ Annotated Checklist of Brazilian Mammals. Occas. Pap. Conserv. Biol. 1-76.

PEDÓ, E. 2005. Assembléia de pequenos mamíferos não-voadores em área de ecótono campo - floresta com Araucária na região dos Campos de Cima da Serra, Rio Grande do Sul. Dissertação de mestrado, Universidade Federal do Rio Grande do Sul, Porto Alegre.

QUINTELA, F.M., SANTOS, M.B., CHRISTOFF, A.U. \& GAVA, A. 2011. Notas sobre morfologia, distribuição geográfica, história natural e citogenética de Cryptonanus guahybae (Didelphimorphia: Didelphidae). Mastozool. Neotrop. 18(2):247-257.

QUINTELA, F.M., SANTOS, M.B., CHRISTOFF, A.U. \& GAVA, A. 2012. Pequenos mamíferos não-voadores (Didelphimorphia, Rodentia) em dois 
fragmentos de matas de restinga de Rio Grande, Planície Costeira do Rio Grande do Sul. Biota Neotrop. 12(1):261-266http://www.biotaneotropica. org.br/v12n1/en/abstract?inventory+bn02412012012 (ultimo acesso em 07/09/2012)

SANTOS, M.B., OLIVEIRA, M.C.L.M. \& TOZETTI, A.M. 2012. diversidade e uso do ambiente por serpentes e lagartos em ambientes costeiros do extremo sul brasileiro. Biota Neotrop. 12(3):78-87. http://www. biotaneotropica.org.br/v12n3/pt/abstract?article+bn01812032012 ISSN 1676-0603 (ultimo acesso em 07/09/2012).

SANTOS, M.B., OLIVEIRA, M.C.L.M., GONÇALVES, T.P., ALMEIDA, F.M., LOEBMANN, D. \& TOZETTI, A.M. 2013. Does human inffluence on coastal grassland habitats affect predation pressure on snakes? Biota Neotrop. 13(1):366-370. http://www.biotaneotropica.org.br/v13n1/en/ abstract?short-communication +bn01913012013 (ultimo acesso em 18/07/2013)

SPONCHIADO, J., MELO, G.L. \& CÁCERES, N.C. 2012. Habitat selection by small mammals in Brazilian Pampas biome. J. Nat. Hist. 46(2122):1321-1335. http://dx.doi.org/10.1080/00222933.2012.655796

VIEIRA, E.F. 1983. Rio Grande: geografia física, humana e econômica. Sagra, Porto Alegre.

VIEIRA, E.F. 1984. Rio Grande do Sul: geografia física e vegetação. Sagra, Porto Alegre.

WAECHTER, J.L. 1985. Aspectos ecológicos da vegetação de restinga no Rio Grande do Sul, Brasil. Comum. Mus. Ciênc. Tecnol. PUCRS, ser. Bot. 33:49-68. 